\title{
Accoucher à la maison
}

\section{Valérie Junod}

Professeure de droit aux Universités de Genève et de Lausanne

Donner naissance chez soi? Pour beaucoup de femmes, cette possibilité paraît très peu attractive, voire douloureuse et dangereuse. Cependant, pour une petite minorité [1], c'est l'assurance d'accoucher de manière «naturelle» dans un cadre familier, avec seulement un minimum d'interventions médicales, tout en garantissant un contact étroit et prolongé avec le nouveau-né.

\section{Les points-clés de l'arrêt européen}

Le 15 novembre 2016, la Grande Chambre de la Cour européenne des droits de l'homme à Strasbourg (ci-après la Cour) a eu à décider si les Etats du Conseil de l'Europe dont la Suisse fait partie - peuvent interdire aux sagesfemmes d'accompagner un accouchement à domicile [2]. En l'occurrence, la République tchèque empêchait les sages-femmes de fournir leurs services ailleurs que dans des hôpitaux dûment équipés et placés sous la direction d'un médecin [3]. Si une femme voulait accoucher à la maison, elle devait le faire seule, ne pouvant obtenir l'assistance ni d'une sage-femme, ni d'un médecin.

Deux mères s'en sont plaintes à la Cour. La première, au terme d'une grossesse normale, a accouché seule à la maison de son deuxième enfant, après avoir tenté en vain de se procurer l'aide d'une sage-femme. La deuxième, également en bonne santé, a dû se résoudre à accoucher à l'hôpital de son troisième bébé, après avoir intenté des démarches similaires tout aussi vaines; auparavant, elle était parvenue à accoucher de ses deux premiers enfants à domicile avec le soutien d'une sage-femme.

\section{Si une femme voulait accoucher à la maison, elle devait le faire seule.}

Une première fois en 2014, une section de la Cour (7 juges) a conclu que l'interdiction tchèque ne violait pas le droit à la vie privée des deux patientes, droit garanti par l'article 8 de la Convention européenne sur les droits de l'homme (CEDH). L'affaire a cependant été jugée suffisamment importante - ce qui est rare (environ $5 \%$ des cas) - pour que la Grande chambre (17 juges) accepte de la revoir.

La Grande chambre parvient cependant à la même conclusion: à une majorité de 12 juges contre 5, elle considère que les Etats jouissent d'une large marge d'appréciation dans ce domaine et que le choix d'interdire tout accompagnement médical pour une naissance à la maison n'outrepasse pas cette marge de manœuvre. Même s'il y a effectivement une atteinte au droit à la vie privée des femmes [4], cette ingérence demeure proportionnée au but poursuivi par l'Etat, à savoir la protection de la santé des nouveau-nés, voire des parturientes. Les Etats n'ont donc pas à aménager leur système de santé pour rendre possible l'accompagnement de l'accouchement à domicile.

Pour soutenir cette conclusion, la Cour avance plusieurs arguments. D'abord, elle retient que les naissances à la maison sont statistiquement plus risquées pour l'en-

Si ce danger peu probable se matérialise, les conséquences individuelles peuvent être très sérieuses.

fant et la mère, comparées à celles intervenant à l'hôpital (à ce sujet, voir cependant le point 3 ci-dessous) [5]. Ensuite, elle estime qu'il n'y a pas de large consensus entre Etats membres en faveur de l'accouchement à domicile [6]. Certes, 20 Etats le permettent expressément, dont 15 qui de surcroît le remboursent par l'assurance sociale [7]. Cependant, dans les autres Etats, l'accouchement à domicile n'est pas explicitement admis, la législation étant le plus souvent muette à ce sujet [8]. La Cour en déduit que la question de savoir si et comment un accouchement à domicile doit être permis, respectivement encadré, est délicate, tant sous l'angle médical, technique qu'économique, de sorte que les législateurs nationaux sont les mieux placés pour décider comment procéder [9]. Dans la pesée entre l'intérêt de la femme à choisir et l'intérêt de l'Etat à protéger la vie et la santé des nouveau-nés [10], la Cour accorde la priorité au second [11].

L'arrêt est accompagné de deux opinions minoritaires (dissidentes) des cinq juges estimant que la législation tchèque viole bel et bien l'article $8 \mathrm{CEDH}$. Ils mettent en exergue l'incongruité du choix - accoucher seule à la maison ou accoucher à l'hôpital - auquel la loi tchèque accule la femme enceinte [12]. Ils relèvent que si le but réellement poursuivi par l'Etat est de protéger la santé des nouveau-nés et des femmes, la préférence devrait être donnée à un accouchement accompagné à 
la maison, plutôt que de contraindre les femmes absolument réfractaires au cadre hospitalier à accoucher sans aucune aide chez elles [13].

\section{Commentaires critiques}

Même si la Suisse n'est pas directement touchée par cet arrêt, puisqu'elle permet et même rembourse l'accouchement à domicile encadré par les sages-femmes, elle demeure concernée par les questions générales importantes qu'il soulève au regard de la santé publique. Nous en mettons ici trois en évidence.

\section{1) La restriction du droit à l'autonomie}

Premièrement, l'arrêt amène à s'interroger sur le pouvoir de l'Etat de restreindre la liberté individuelle (le droit à l'autonomie) en présence d'un risque médical de faible probabilité.

Dans le cas d'une grossesse à bas risque, un accouchement à la maison suivi par une sage-femme est considéré statistiquement peu risqué (pour la notion de bas risque ainsi que pour le détail des études, voir le point 3 ci-dessous). Cependant, si ce danger peu probable se matérialise, les conséquences individuelles peuvent être très sérieuses, allant jusqu'au décès de la mère et/ou de l'enfant. D'où la question: est-ce qu'un risque statistiquement minime d'un atteinte parfois grave à la santé justifie qu'on prive l'individu de son droit de choisir?

Dans la quasi-totalité des domaines médicaux, la réponse est clairement non. Le patient peut refuser une intervention qui pourrait sauver sa vie, comme il peut accepter une intervention dotée d'une faible chance de succès et d'un risque élevé pour sa vie [14].

\section{2) La protection des nouveau-nés}

Ceci amène directement à la deuxième interrogation connexe: ce pouvoir de l'Etat de restreindre la liberté individuelle doit-il être plus étendu lorsque les personnes à protéger sont des fœtus in utero ou des bébés en train de naître?

Cette question est éminemment sensible. Aux EtatsUnis notamment [15], des dizaines de femmes enceintes sont, chaque année, condamnées à la prison ou subissent des interventions médicales forcées au motif que leurs choix de vie sont jugés nocifs pour la santé de leurs bébés in utero; elles sont punies par la justice notamment pour avoir refusé une césarienne ou un autre suivi médical, pour avoir consommé des drogues ou de l'alcool ou pour vouloir accoucher à la maison contre l'avis des médecins [16].

En tout cas en Europe, on conçoit mal un système où l'Etat serait habilité à régenter la vie des femmes en- ceintes en leur interdisant toute prise de risques: peu de femmes seraient d'accord [17] qu'on leur interdise pendant neuf mois toute consommation d'alcool ou de cigarettes; qu'on les force au régime lorsqu'elles sont en surpoids; qu'on leur interdise les sports jugés dangereux; qu'on les place au repos forcé lorsque cela peut retarder une naissance qui s'annonce prématurée. Un tel système reviendrait à placer sous tutelle un large pan de la population - à supposer au demeurant que cela soit faisable sur un plan pratique. De surcroît, si l'on devait approuver ce sacrifice de la liberté individuelle en faveur des enfants à naître, qu'est-ce qui empêcherait d'imposer ensuite des sacrifices analogues en faveur des enfants nés ou même d'autres personnes au sein de la famille?

Rejeter l'intervention de l'Etat dans la vie quotidienne des femmes enceintes est la position éthiquement juste. En effet, il est délicat de fixer une limite rationnelle à une telle intervention [18]. De plus, celle-ci risque de détourner les femmes enceintes d'avoir recours au système de santé, de peur d'être exposées

La probabilité que l'un ou l'autre de ces risques se matérialise dépend fortement de circonstances que les études ont pu identifier.

(directement ou indirectement) à des sanctions, faisant ainsi peser sur l'enfant à naître une menace encore plus lourde. Dès lors, seul une probabilité élevée et établie de manière fiable d'un dommage sérieux pour le fœtus peut légitimer une intervention, et ce pour autant que le choix de la femme ne lui procure pas, à elle, un bénéfice prépondérant. De surcroît, l'intervention concrètement envisagée par l'Etat doit avoir des chances élevées d'atteindre le but qui lui est attribué, sans qu'une mesure moins incisive ne puisse entrer en ligne de compte. Tant que ces conditions ne sont pas clairement remplies, c'est la liberté individuelle qui doit l'emporter.

\section{3) L’appréciation des études médicales}

La troisième interrogation a trait à l'évaluation par la Cour des études médicales.

L'arrêt relève les très bons résultats obtenus par la République tchèque en matière de mortalité des nouveaunés. En effet, la République ne dénombre que 1,7 décès (dans les 27 premiers jours de vie) pour 1000 naissances [19]. Cependant, il est à souligner que ce taux très bas ne peut pas être attribué causalement à l'interdiction des accouchements accompagnés à domicile [20]; d'autres Etats font d'ailleurs état de taux tout aussi bas et admettent de tels accouchements [21].

De nombreuses études ont tenté de répertorier et de quantifier les divers risques d'un accouchement à 


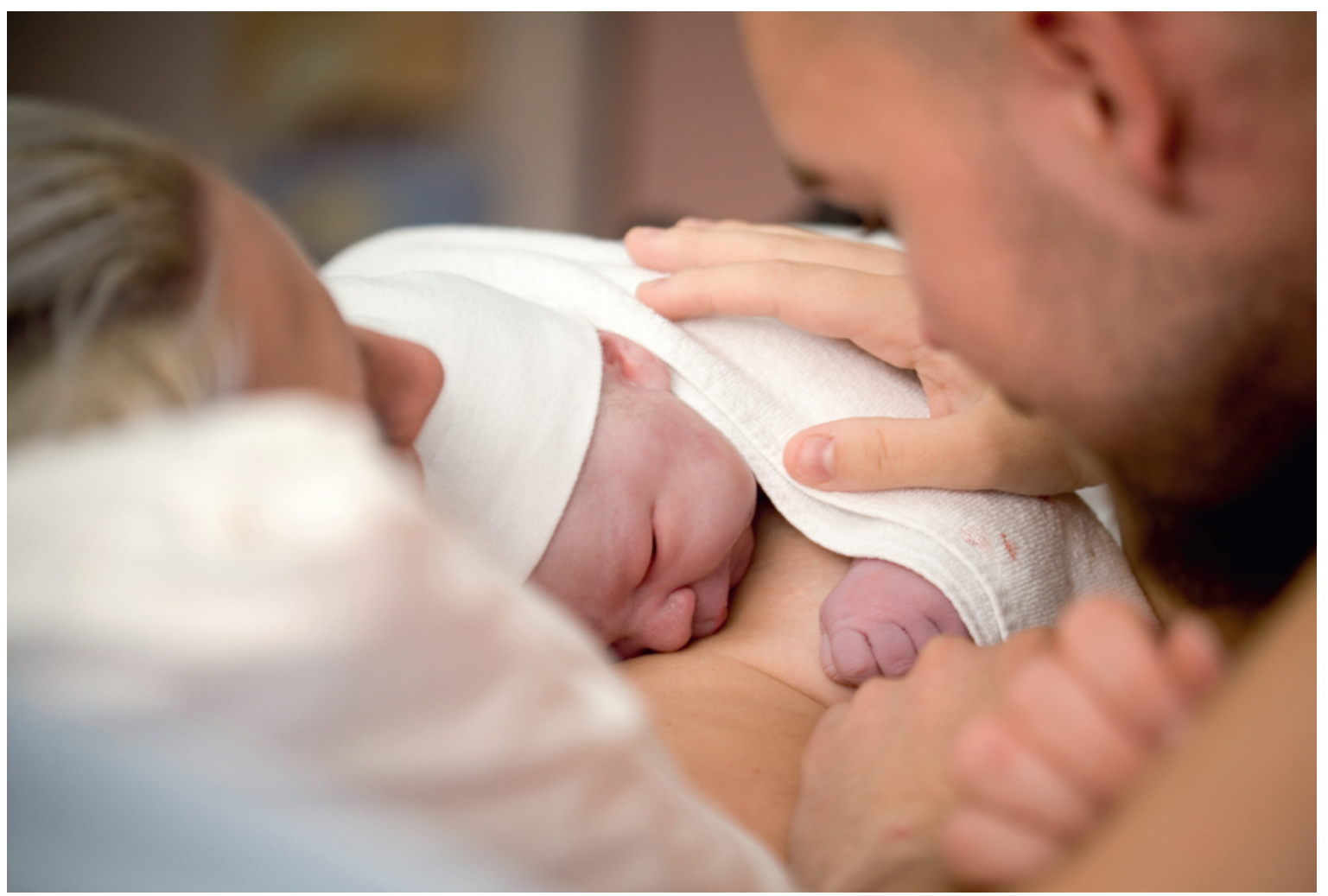

Une nouveau-née avec ses parents.

domicile. Malheureusement, l'arrêt ni ne cite, ni a fortiori ne commente ces études - d'où une perspective biaisée des risques liés à l'accouchement à domicile. Certes, les résultats des études ne sont de loin pas tous concordants [22]. Font de surcroît défaut des essais cliniques randomisées, par définition plus fiables [23]; aussi, les études disponibles ne peuvent établir qu'une corrélation, et non un lien de cause à effet [24]. S'agissant du risque de décès périnatal lors d'accouchements à domicile décidés dans des cas qualifiés de bas risque, certaines études font état d'un risque doublé, voire triplé [25], tandis que d'autres concluent à un risque équivalent, voire réduit [26]; en toute hypothèse, le risque absolu de décès périnatal dans les pays riches reste bas, généralement de l'ordre de 1 pour 1000 naissances. Le même constat ambivalent s'applique au risque de dommages à long terme ou de complications sérieuses pour le nouveau-né [27].

Si le doute subsiste quant à la sécurité pour l'enfant né à la maison, les études disponibles sont en revanche largement unanimes pour observer un risque sensiblement moindre d'interventions médicales sur le corps de la femme [28] (induction, épisiotomie, naissance aux forceps, monitoring du rythme cardiaque, césarienne) lors d'accouchements à domicile. Le risque de dommages médicaux subis par la mère (notamment déchirures vaginales accidentelles, infections, hémorragies) serait plus faible également.

Le constat qui se dégage de ces études est que l'accouchement à la maison présente à la fois des avantages relativement certains et des risques moins clairement établis. Point important: la probabilité que l'un ou l'autre de ces risques se matérialise dépend fortement des circonstances que les études ont pu identifier. Le cas de figure considéré idéal pour un accouchement à domicile est le suivant: la mère a déjà accouché par voie basse d'un enfant né en bonne santé; elle-même est en bonne santé; elle a moins de 35 ans; sa grossesse actuelle n'a rencontré aucun problème et a fait l'objet d'un suivi médical régulier; la future mère a planifié son accouchement à domicile avec le soutien d'une sage-femme dûment formée et expérimentée [29]; le fœtus (unique) est en bonne santé et se présente en position céphalique; l'accouchement a lieu entre la $37^{\mathrm{e}}$ et la $41^{\mathrm{e}}$ semaine de grossesse; le domicile se situe non loin d'un hôpital, lequel a été prévenu d'un possible transfert en cas de complications et s'est organisé en conséquence. Lorsque ces «conditions-cadre» optimales sont réunies, l'accouchement à domicile représente une option globalement sûre, voire plus sûre que l'accouchement à l'hôpital. C'est pourquoi le NICE anglais (National Institute for Health and Care Excellence) constate: «the evidence 
Les références se trouvent sous www.bullmed.ch $\rightarrow$ Numéro actuel ou

$\rightarrow$ Achives $\rightarrow 2017$

$\rightarrow$ Numéro 2017/32.

Correspondance: Prof. Dr Valérie Junod Université de LausanneHEC Anthropole bureau 3076

CH-1015 Lausanne valerie.junod[at]unil.ch now shows midwife-led units to be safer than hospital for women having a straightforward (low risk) pregnancy. [...] home birth is equally as safe as a midwifeled unit and traditional labour ward for the babies of low risk pregnant women who have already had at least one child previously". [30]

A mon sens, ce constat devrait amener à une plus grande prudence du législateur et notamment une plus grande réticence à vouloir dicter son choix aux patientes. Lorsque les études font état de risques pour la santé globalement équivalents dans une alternative comme dans l'autre, il faudrait en conclure - sur le plan juridique - que l'Etat n'a pas prouvé la nécessité de son ingérence dans la vie privée. Dès lors, la liberté de l'individu devrait primer sur l'intérêt public à protéger la vie du nouveau-né. C'est d'ailleurs la position défendue par bon nombre des comités d'éthique et groupements professionnels [31]. La Cour aurait dû analyser plus soigneusement les données scientifiques ainsi que les prises de position éthiques.

\section{Conclusion}

Il est regrettable que la Cour européenne des droits de l'homme ait refusé, dans sa pesée des intérêts, d'accorder aux femmes la faculté de choisir les risques qu'elles veulent assumer pour elles et pour leurs enfants à naître. Comme le concède l'arrêt, donner naissance est un des aspects les plus intimes de la vie d'une femme.
C'est le point de départ de responsabilités constantes que la mère assumera pendant les longues années où elle élèvera son enfant. La très grande majorité des femmes assument avec sérieux et amour ces responsabilités. Ne serait-ce qu'en reconnaissance de ce dévouement, l'Etat devrait s'abstenir d'imposer sa solution [32].

Bien sûr, cela n'empêche pas que le choix des modalités d'accouchement fasse l'objet d'un encadrement, y compris juridique, approprié. Il revient d'abord aux milieux médicaux d'indiquer les circonstances dans

\section{C'est le point de départ de responsabilités} constantes que la mère assumera pendant les longues années où elle élèvera son enfant.

lesquelles un accouchement à domicile peut s'effectuer selon les règles de l'art [33]. Ensuite, ces recommandations médicales peuvent être entérinées par le législateur, tant sur le plan administratif (octroi des autorisations de pratique aux professionnels de la santé) que sur le plan civil (responsabilité en cas de non-respect). Finalement, en matière de santé, y compris de santé publique, c'est par le dialogue entre professionnels de la santé et patients que les solutions optimales à long terme peuvent se dégager.

\section{Crédit photo}

(c) Molka| Dreamstime.com 
Notes

1 Le pourcentage de femmes qui accouchent (de manière planifiée) à la maison est d'environ 1\% en Suisse. Cf. Zeynep Ersan Berdoz, Accoucher à domicile? Entre crainte et méconnaissance, Sage-femme suisse $12 / 2004$, p. 28.

2 Affaire Dubská et Krejzová c. République tchèque $\left(\mathrm{n}^{\text {os }} 28859 / 11\right.$ et 28473/12). Dans une précédente affaire Ternovszky du 14 décembre 2010, la Cour avait condamné la Hongrie pour n'avoir pas mis en place de réglementation précise encadrant l'accouchement à domicile, ce qui avait pour conséquence que cette option était, dans la pratique, quasi-exclue. La Cour avait alors estimé qu'une telle ingérence dans la vie privée des femmes devait impérativement reposer sur une réglementation étatique claire et prévisible ce qui n'était pas le cas en Hongrie.

3 La législation tchèque était remarquablement peu claire et donc difficile à interpréter, ce que les juges majoritaires et minoritaires ont incidemment relevé. Consid. 171 de l'arrêt et consid. 2 de l'opinion minoritaire.

4 Cf. consid. 185. Dans la précédente affaire Ternovszky de 2010, la Cour avait déjà dégagé la conclusion que le choix par les femmes des modalités de leur accouchement relève bel et bien de la protection de la vie privée protégée par l'article 8 CEDH (consid. 22). Dès lors, cette liberté ne peut être réduite que si l'Etat peut invoquer une base légale et un intérêt public supérieur, tout en respectant le principe de la proportionnalité.

5 Consid. 185 et 186 de l'arrêt commenté.

6 Consid. 183.

7 Consid. 67 et 68 de l'arrêt commenté.

8 Pour les juges minoritaires, le fait que seuls quatre Etats aient légiféré pour interdire explicitement l'accouchement à domicile est au contraire signe d'un consensus européen en faveur d'une telle possibilité. Cf. consid. 28 de l'opinion minoritaire en lien avec consid. 99 de l'arrêt.

9 Consid. 177 à 184

10 Pourtant, la Cour a toujours refusé de reconnaître aux fœtus in utero les droits garantis par la CEDH. Cf. affaire Vo c. France du 8 juillet 2004. Elle s'est aussi abstenue de trancher pour ou contre un droit à avorter directement tiré de la CEDH. Cf. Grégor Puppinck, L'avortement et la CEDH, Mélanges en l'honneur de Gérard Mémeteau, Droit médical et éthique médicale: regards contemporains, Ed. Les Etudes hospitalières, 2015.

11 La majorité de la Grande chambre conclut toutefois avec une invitation adressée à la République tchèque à réexaminer la nécessité de son interdiction en fonction des nouvelles connaissances médicales qui viendraient à être disponibles. Elle encourage également l'Etat mis en cause à améliorer les conditions dans lesquelles les femmes donnent naissance dans les hôpitaux, de nombreuses critiques ayant été formulées quant aux modalités d'accouchement peu respectueuses de l'autonomie des patientes (notamment des interventions médicales sans consentement libre et éclairé; des contraintes imposées dans la manière d'accoucher; une obligation de demeurer au moins trois jours à l'hôpital). Voir aussi la précédente condamnation de la République tchèque dans l'affaire Hanzelkovi tranchée le 11 décembre 2014.

12 Consid. 8 de l'opinion minoritaire.

13 On ajoutera que ces femmes peuvent être amenées à minimiser leurs interactions avec les médecins et l'hôpital déjà pendant leur grossesse, si elles sont conscientes que leur projet d'accoucher à domicile peut déboucher sur des sanctions à l'encontre de leur sage-femme, ce dans l'espoir que les autorités restent dans l'ignorance de leur projet. Voir aussi les remarques au consid. $138 \mathrm{de}$ l'arrêt commenté.

14 La question de savoir si l'assurance sociale doit ou non rembourser de telles prestations est une question distincte, dont il n'est pas question ici.

15 Mais voir également en Europe l'affaire Hanzelkovi c. République tchèque déjà mentionné, où une mère a été ramenée de force à l'hôpital, car le médecin et un tribunal avaient jugé qu'un nouveau-né doit rester à l'hôpital au moins les 3 jours qui suivent sa naissance.

16 Lynn M. Paltrow et Jeanne Flavin ont répertorié 413 affaires où, entre 1973 et 2005, des femmes américaines ont été poursuivies par la justice en raison des risques (perçus) de leur conduite pour leur grossesse. Leur passionnant article résume les aspects saillants de ces différentes affaires, mettant notamment en évidence la discrimination raciale sous-jacente. Cf. Arrests and forced interventions on pregnant women in the United States, 1973-2005: Implications for Women's legal status and public health, Journal of Health Politics, Policy and Law, 2013. Voir aussi Stephen W. Patrick \& Davida M. Schiff. A Public Health Response to Opioid Use in Pregnancy. Policy Statement. American Academy of Pediatrics, 139 Pediatrics e20164070 (2017).

17 On pense par exemple à la femme qui entreprendrait un traitement pharmaceutique contre son cancer pour augmenter ses chances de guérir, quand bien même le médicament pourrait nuire à la santé de son fœtus.

18 On rappellera qu'en matière d'avortement en Suisse, la femme enceinte est libre de son choix pendant les 12 premières semaines d'aménorrhée. Après cette date, elle peut avorter à condition que l'interruption de grossesse soit «nécessaire pour écarter le danger d'une atteinte grave à l'intégrité physique ou d'un état de détresse profonde de la femme enceinte». Ces normes du Code pénal (art. 119 principalement, accessoirement art.120) délimitent la protection due à l'embryon et au fœtus in utero. Cf. généralement Dominique Manaï, L'embryon face au droit: une entité polymorphe à géométrie variable, Jusletter 19 janvier 2009. Par ailleurs, en matière de recherche médicale, une femme enceinte peut participer à une étude laissant espérer un bénéfice direct à condition que «le rapport entre les risques et les contraintes prévisibles pour la femme enceinte, pour l'embryon ou le fœtus, d'une part, et le bénéfice escompté, d'autre part, [ne soit] pas disproportionné.» (art. 26 de la Loi sur la recherche sur l'être humain). Il faut attendre la naissance de l'enfant vivant pour que celui-ci jouisse de la pleine protection accordée par le droit aux personnes (art. 31 Code civil). En particulier, les dispositions du Code civil et du Code pénal protégeant son intégrité physique et sa personnalité lui sont applicables, même si l'art. $116 \mathrm{CP}$, disposition quelque peu désuète, punit moins sévèrement la mère qui a tué (homicide) son enfant «sous l'influence de l'état puerpéral».

19 Voir les consid. 28 et 29.

20 Le pourcentage de naissances à domicile est de toute façon très bas ( $1 \%$ environ) dans la majorité des pays d'Europe, à l'exception des Pays-Bas. Il faudrait donc que les naissances à domicile soient associées à un nombre significativement plus élevé de décès pour que cette différence puisse se refléter dans les taux globaux de mortalité.

21 Cf. consid. 21 de l'opinion dissidente.

22 Cf. American College of Obstetricians and Gynecologists' Committee on Obstetric Practice on Planned Home Birth, Opinion Number 669, August 2016, http://www.acog.org/Resources-And-Publications/Committee-Opinions/Committee-on-Obstetric-Practice/ Planned-Home-Birth.

23 La randomisation est difficile à mettre en œuvre, peu de femme étant disposées à être attribuées de manière aléatoire à l'un ou l'autre des bras de l'étude. C'est pourquoi la grande majorité des études disponibles sont des études observationnelles ou des études rétrospectives, notamment de registres. De telles études sont par nature moins fiables. Cependant, les études menées s'efforcent toutes de minimiser les biais, notamment en répertoriant les paramètres ("outcomes») observés en fonction de l'intention de la femme quant au lieu d'accouchement, et non pas en fonction du lieu où l'enfant est finalement né.

24 Les femmes qui choisissent d'accoucher à domicile présentent souvent des caractéristiques différentes de la population des parturientes en général. Souvent, elles appartiennent à des couches socio-économiques favorisées. Cf. Ursula Ackermann-Liebrich et al., Home versus hospital deliveries: follow up study of matched pairs for procedures and outcome. Zurich Study Team, 313 BM p.1313-8, 1996; Helen Hazen, The First Intervention is Leaving Home": Reasons for Electing an Out-of-hospital Birth among Minnesotan Mothers, Medical Anthropology Quarterly, accepted for publication 2017; Mickey Sperlich et al., Where do you feel safest? Demographic factors and place of births, Journal of Midwifery \& Women's Health, p. 88, 2016.

25 Cf. Jonathan M. Snowden et al., Planned out-of-hospital birth outcomes, 373 New England Journal of Medicine p. 2642-2653, 2015. Amos Grünebaum et al., Planned home births: the need for additional contraindications, American Journal of Obstetrics \& Gynecology (AJOG), p. 401.e1-27 2017; Wax et al., Maternal and newborn outcomes in planned home birth vs. planned hospital births: a 
metaanalysis, 203(3) AJOG, p. 243.e1-243.e8, 2010, étude critiquée par Russell S. Kirby et Jordana Frost, ACOJ p.e16, 2011; Kennare et al., Planned home and hospital births in South Australia, 19912006: differences in outcomes, 192 The Medical Journal of Australia p. $76-802010$

26 Cf. Ackermann-Liebrich et al., déjà cité sous $n^{\circ} 1$; Patricia $\mathrm{A}$. Janssen et al., Outcomes of planned home birth with registered midwife versus planned hospital birth with midwife or physician, 181 Canadian Medical Association Journal (CMAJ) p. 377-83, 2009; Eileen K. Hutton et al., Outcomes associated with planned home and planned hospital births in low-risk women attended by midwives in Ontario, Canada, 2003-2006: a retrospective cohort study, 36(3) Birth p. 180-9, 2009; Janssen et al., Outcomes of planned hospital birth attended by midwives compared with physicians in British Columbia, 34(2) Birth: p. 140-7, 2007; Janssen et al., Outcomes of planned home births versus planned hospital births after regulation of midwifery in British Columbia, $166 \mathrm{CMAJ}$ p. 315-23, 2002.

27 Pour un récapitulatif clair et complet de 15 études de cohorte, voir Heather R. Elder et al., Investigating the debate of home birth safety: A critical review of cohort studies focusing on selected infant outcomes, 13 Japan Journal of Nursing Science p. 297-308 (2016).

28 Cf. Snowden et al., Janssen, cité supra nnn, Birth 2007; Janssen, CMAJ 2009.

29 Cf. notamment Cheng et al., Selected perinatal outcomes associated with planned home births in the United States, 209 AJOC p. 325.e1-8, 2013

30 Communiqué de presse du 3 décembre 2014, intitulé «NICE confirms midwife-led care during labour is safest for women with straightforward pregnancies». Plus précisément, la recommandation du NICE est: «Advise low risk multiparous women that plan- ning to give birth at home or in a midwifery led unit (freestanding or alongside) is particularly suitable for them because the rate of interventions is lower and the outcome for the baby is no different compared with an obstetric unit. Advise low risk nulliparous women that planning to give birth in a midwifery led unit (freestanding or alongside) is particularly suitable for them because the rate of interventions is lower and the outcome for the baby is no different compared with an obstetric unit. Explain that if they plan birth at home there is a small increase in the risk of an adverse outcome for the baby.» Dans une étude de suivi effectuée en février 2015, le NICE a relevé que seulement $41 \%$ des femmes se sont vu expressément offrir le choix du lieu d'accouchement. Cf. NICE, page web intitulée «Intrapartum care: care of healthy women and their babies during childbirth [CG190], Measuring the use of this guidance». La recommandation complète est accessible à partir de https://www.nice.org.uk/guidance/cg190/.

31 Pour un descriptif des recommandations professionnelles pour et contre la naissance à domicile, voir l'intéressante étude de S. Roome et al., Why such differing stances? A review of position statements on home birth from professional colleges, BJOG p. 376-83, 2016.

32 Comme l'écrivent les juges minoritaires, «there is generally no conflict of interest between the mother and her child. [...] under ordinary circumstances, we trust that a mother will choose the best option for the birth of her child.» Consid. 7.

33 Dans les affaires Kück c. Allemagne de 2003 et Schlumpf c. Suisse de 2009, la Cour avait pourtant jugé que lorsque l'issue d'un litige dépend avant tout de l'appréciation de données médicales (ici la nécessité d'une opération de conversion sexuelle), le législateur et la justice sont tenus de respecter l'avis des experts médicaux, sans être habilités à poser des exigences générales plus strictes et non fondées sur la science. 